\title{
Bilateral Anterior Subcapsular Cataract Development Following Initiation of Enfortumab Vedotin
}

\section{Alexa Thibodeau (id \\ Nambi Nallasamy (ID)}

Department of Ophthalmology and Visual Sciences, W.K. Kellogg Eye Center, University of Michigan, Ann Arbor, MI, USA
Correspondence: Nambi Nallasamy Department of Ophthalmology and Visual Sciences, W.K. Kellogg Eye Center, University of Michigan, 1000 Wall Street, Ann Arbor, MI, 48105, USA

Tel +I 7347635506

Fax +I 7349362340

Email nnallasa@med.umich.edu

\begin{abstract}
Enfortumab vedotin is an antibody-drug conjugate that was recently granted accelerated US Food and Drug Administration approval for the treatment of locally advanced or metastatic urothelial cancer. Early clinical trials identified blurry vision, increased lacrimation and other events associated with dry eye as potential side effects. We report a case of bilateral anterior subcapsular cataract development following initiation of enfortumab vedotin. Enfortumab vedotin is not previously known to cause cataract development or progression and, thus, our patient's presentation may reflect the first report of an undocumented adverse effect of this novel agent.
\end{abstract}

Keywords: anterior subcapsular cataract, enfortumab vedotin, urothelial carcinoma

\section{Introduction}

Cataract is the leading cause of reversible blindness in the world. ${ }^{1}$ Aging, genetics, systemic disease, trauma, inflammation and toxic exposure are all known contributors to cataract development, with strong associations for particular cataract subtypes. ${ }^{2}$ Commonly classified by location and morphology of the lens opacity, cataract subtypes include nuclear, cortical, subcapsular and combined. Anterior subcapsular cataract formation results from fibrous metaplasia of the lens epithelium and is associated with ocular trauma, inflammation or irritation. Cataracts may cause varying degrees of visual impairment depending on location and severity. When a patient develops a significant decline in visual functioning or reduction in quality of life, surgical intervention may be considered.

In December 2019, the US Food and Drug Administration granted accelerated approval for enfortumab vedotin, a Nectin-4 targeting antibody-drug conjugate indicated for the treatment of adult patients with locally advanced or metastatic urothelial cancer. We report a case of visually significant bilateral anterior subcapsular cataract development following therapy with enfortumab vedotin. While the exact mechanism remains to be elucidated, patients and physicians should be aware of the risk of anterior subcapsular cataract formation following enfortumab vedotin initiation.

\section{Case Description}

A 62-year-old male with a history of metastatic urothelial carcinoma was enrolled in NCT03288545, a phase $1 \mathrm{~b}$ national clinical trial designed to examine the safety, 


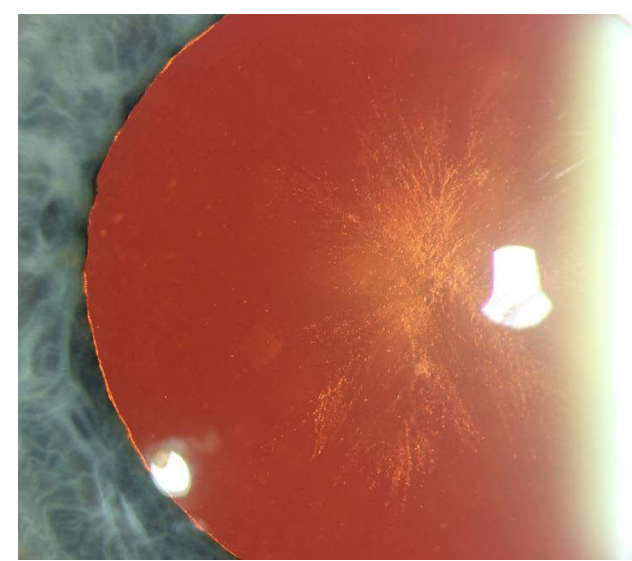

Figure I Retroillumination slit-lamp photograph highlighting the anterior subcapsular opacification of the crystalline lens involving the visual axis of the left eye after 18 months of therapy with enfortumab vedotin.

tolerability and efficacy of enfortumab vedotin alone and in combination with other anticancer therapies. The patient was initiated on enfortumab vedotin in combination with cisplatin. The study began on October 11, 2017, and is estimated to run until December $31,2026 .^{3}$ The patient had no past ocular history and baseline eye exam demonstrated a best-corrected visual acuity of 20/20 in both eyes. He was noted to have transparent crystalline lenses and an unremarkable anterior and posterior segment examination. While undergoing treatment, the patient noticed a gradual decline in his vision. Repeat examination 18 months after initiation of therapy demonstrated a two-line decrease in best-corrected visual acuity that was further reduced to 20/ 60 and 20/200, respectively, upon glare testing. Slit-lamp examination revealed interval development of bilateral, symmetric 2-3+ anterior subcapsular and 1+ posterior subcapsular cataracts (Figure 1). Over this same time frame, the patient received intermittent low-dose (5$10 \mathrm{mg}$ ) oral prednisone in addition to systemic dexamethasone with his chemotherapy infusions. Both enfortumab vedotin and cisplatin were administered at their standard recommended doses. He had no history of radiation therapy or diabetes. After discussion of options regarding his visually significant cataracts, the patient elected to proceed with phacoemulsification and intraocular lens implantation for both eyes. Post-operatively, he noted significant improvement in his vision and best-corrected visual acuity measured 20/20 for each eye.

\section{Discussion}

Enfortumab vedotin directly targets Nectin-4, an adhesion protein highly expressed in bladder cancer cells. Following endocytosis, the complex releases monomethyl auristatin E, a microtubule-disrupting agent that induces cell cycle arrest and, ultimately, apoptotic cell death. ${ }^{4}$ Safety data from Phase I and Phase II clinical trials prompted early warnings of the possibility of ocular disorders with enfortumab vedotin therapy. ${ }^{4,5}$ In these studies, $46 \%$ of the 310 patients treated developed ocular side effects. ${ }^{6}$ Most events were attributable to ocular surface disease, with dry eye symptoms reported in $36 \%$ of patients and blurred vision in $14 \%$. There was a median time of 1.9 months to symptom onset and no report of cataract development. ${ }^{4}$ Since approval in 2019 , there have been multiple case reports of adverse cutaneous reactions related to enfortumab vedotin use; however, there have been no published reports of adverse ophthalmic effects attributed to the drug. ${ }^{7-10}$

Anterior subcapsular cataract results from fibrous metaplasia of the lens epithelium. This type of lens opacification is associated with ocular trauma, inflammation or irritation; atopic dermatitis, electrical burn and irradiation are typical scenarios. ${ }^{11}$ Our patient did not manifest any clinical signs or conditions known to be associated with anterior subcapsular cataract development. Additionally, cisplatin, our patient's only other new medication exposure, has been in medical use for over four decades and is not associated with cataract formation. His intermittent systemic steroid use likely contributed to the development of the posterior subcapsular component of his cataracts. Steroids are a well-established risk factor for posterior subcapsular cataracts but not for anterior subcapsular cataracts.

To the best of our knowledge, this is the first reported case of anterior subcapsular cataract formation as a consequence of enfortumab vedotin therapy. Histologic evidence shows that transforming growth factor- $\beta$ (TGF- $\beta$ ) plays a critical role in lens epithelial cell proliferation and epithelialmesenchymal transition. Inappropriate TGF- $\beta$ signaling with subsequent accumulation of extracellular matrix is strongly implicated in anterior subcapsular cataract pathogenesis. ${ }^{12}$ It is possible that enfortumab vedotin crossreactivity with Nectin-3, which is expressed in the anterior epithelial cells of the crystalline lens, may contribute to this process. ${ }^{13}$ Although not routinely performed, utilization of immunohistochemical staining to detect Nectin-4 and Nectin-3 expression on the anterior lens capsular tag could help elucidate the mechanism of action for anterior subcapsular cataract formation. Given the irreversible nature of cataracts and the need for surgical intervention to restore vision, pre-treatment counseling regarding this adverse effect of enfortumab vedotin is warranted. 


\section{Conclusion}

Blurry vision, dry eye and anterior subcapsular cataract development are potential adverse effects of enfortumab vedotin. A baseline eye exam is recommended for all patients who start enfortumab vedotin. Patients should monitor for vision changes while undergoing therapy.

\section{Ethics Statement}

Institutional review board approval was not required for publication of this case. Written informed consent was obtained from the patient for the case details and the accompanying image to be published.

\section{Disclosure}

The authors report no conflicts of interest in this work.

\section{References}

1. Flaxman SR, Bourne RRA, Resnikoff S, et al. Global causes of blindness and distance vision impairment 1990-2020: a systematic review and meta-analysis. Lancet Glob Health. 2017;5(12):e1221e1234.

2. Gupta VB, Rajagopala M, Ravishankar B. Etiopathogenesis of cataract: an appraisal. Indian $J$ Ophthalmol. 2014;62(2):103-110. doi:10.4103/0301-4738.121141

3. A Study of Enfortumab Vedotin alone or with other therapies for treatment of urothelial cancer (EV-103). NIH: U.S. National Library of Medicine. ClinicalTrials.gov. Available from: https://www.clinical trials.gov/ct2/show/NCT03288545 term $=\mathrm{EV}-103 \&$ draw $=2 \&$ rank $=1$. Accessed June 25, 2021.
4. Chang E, Weinstock C, Zhang L, et al. FDA approval summary: enfortumab vedotin for locally advanced or metastatic urothelial carcinoma. Clin Cancer Res. 2020. doi:10.1158/1078-0432.ccr-202275

5. Rosenberg JE, O’Donnell PH, Balar AV, et al. Pivotal trial of enfortumab vedotin in urothelial carcinoma after platinum and anti-programmed death 1/programmed death ligand 1 therapy. J Clin Oncol. 2019;37(29):2592-2600. doi:10.1200/JCO.19.01140

6. PADCEV (enfortumab vedotin-ejfv) [package insert]. Northbrook, IL: Astellas Pharma US, Inc; 2019.

7. Viscuse PV, Marques-Piubelli ML, Heberton MM, et al. Case report: enfortumab vedotin for metastatic urothelial carcinoma: a case series on the clinical and histopathologic spectrum of adverse cutaneous reactions from Fatal Stevens-Johnson Syndrome/toxic epidermal necrolysis to dermal hypersensitivity reaction. Front Oncol. 2021;11:621591.

8. Sasaki R, Fujimura T, Lyu C, Aiba S. Severe eczematoid and lichenoid eruption with full-thickness epidermal necrosis developing from metastatic urothelial cancer treated with enfortumab vedotin. $J$ Dermatol. 2020;47(12):1436-1438. doi:10.1111/1346-8138.15577

9. Dobry AS, Virgen CA, Hosking AM, et al. Cutaneous reactions with enfortumab vedotin: a case series and review of the literature. JAAD Case Rep. 2021;14:7-9. doi:10.1016/j.jdcr.2021.05.020

10. Francis A, Jimenez A, Sundaresan S, Kelly B. A rare presentation of enfortumab vedotin-induced toxic epidermal necrolysis. JAAD Case Rep. 2021;7:57-59. doi:10.1016/j.jdcr.2020.10.020

11. Bowling B. Kanski's Clinical Ophthalmology: A Systematic Approach. 8th ed. Elsevier; 2016.

12. de Iongh RU, Wederell E, Lovicu FJ, McAvoy JW. Transforming growth factor-beta-induced epithelial-mesenchymal transition in the lens: a model for cataract formation. Cells Tissues Organs. 2005;179 (1-2):43-55. doi:10.1159/000084508

13. Lachke SA, Higgins AW, Inagaki $M$, et al. The cell adhesion gene PVRL3 is associated with congenital ocular defects. Hum Genet. 2012;131(2):235-250. doi:10.1007/s00439-011-1064-z
International Medical Case Reports Journal

\section{Publish your work in this journal}

The International Medical Case Reports Journal is an international, peer-reviewed open-access journal publishing original case reports from all medical specialties. Previously unpublished medical posters are also accepted relating to any area of clinical or preclinical science. Submissions should not normally exceed 2,000 words or 4 published pages including figures, diagrams and references. The manuscript management system is completely online and includes a very quick and fair peer-review system, which is all easy to use. Visit http://www.dovepress.com/testimonials.php to read real quotes from published authors. 